\title{
Integration of Proteomics and Metabolomics in Exploring Genetic and Rare Metabolic Diseases
}

\author{
Michele Costanzo $^{a}$ Miriam Zacchiab Giuliana Bruno ${ }^{c}$ Daniela Criscia, d \\ Marianna Caterino ${ }^{a, d, e}$ Margherita Ruoppolo ${ }^{a, d}$, e \\ aDipartimento di Medicina Molecolare e Biotecnologie Mediche, Università degli Studi di Napoli "Federico II," \\ Naples, b Prima Divisione di Nefrologia, Dipartimento di Scienze Cardio-Toraciche e Respiratorie, Università degli \\ studi della Campania "Luigi Vanvitelli," Scuola di Medicina, 'IRGS Biogem, Ariano Irpino, dCEINGE - Biotecnologie \\ Avanzate scarl, and ${ }^{\mathrm{e} A s s o c i a z i o n e ~ c u l t u r a l e ~ D i S c i M u S ~ R C F, ~ N a p l e s, ~ I t a l y ~}$
}

\section{Keywords}

Acylcarnitines - Biomarkers - Inborn errors of metabolism . Metabolomics $\cdot$ Proteomics

\begin{abstract}
Background: Inherited metabolic disorders or inborn errors of metabolism are caused by deficiency of enzymatic activities in the catabolism of amino acids, carbohydrates, or lipids. These disorders include aminoacidopathies, urea cycle defects, organic acidemias, defects of oxidation of fatty acids, and lysosomal storage diseases. Inborn errors of metabolism constitute a significant proportion of genetic diseases, particularly in children; however, they are individually rare. Clinical phenotypes are very variable, some of them remain asymptomatic, others manifest metabolic decompensation in neonatal age, and others encompass mental retardation at later age. The clinical manifestation of these disorders can involve different organs and/or systems. Some disorders are easily managed if promptly diagnosed and treated, whereas in other cases neither diet, vitamin therapy, nor transplantation appears to prevent multi-organ impairment. Summary: Here, we discuss the principal challenges of metabolomics and proteomics in inherited metabolic disorders. We review
\end{abstract}

\section{KARGER}

(C) 2017 S. Karger AG, Basel

E-Mail karger@karger.com

www.karger.com/kdd the recent developments in mass spectrometry-based proteomic and metabolomic strategies. Mass spectrometry has become the most widely used platform in proteomics and metabolomics because of its ability to analyze a wide range of molecules, its optimal dynamic range, and great sensitivity. The fast measurement of a broad spectrum of metabolites in various body fluids, also collected in small samples like dried blood spots, have been facilitated by the use of mass spectrometry-based techniques. These approaches have enabled the timely diagnosis of inherited metabolic disorders, thereby facilitating early therapeutic intervention. Due to its analytical features, proteomics is suited for the basic investigation of inborn errors of metabolism. Modern approaches enable detailed functional characterization of the pathogenic biochemical processes, as achieved by quantification of proteins and identification of their regulatory chemical modifications. Key Message: Mass spectrometry-based "omics" approaches most frequently used to study the molecular mechanisms underlying inherited metabolic disorders pathophysiology are described.

(c) 2017 S. Karger AG, Basel

Contribution from the International Conference: The Kidney in Genetic and Rare Diseases, Naples, October 27-29, 2016.
Margherita Ruoppolo

Dipartimento di Medicina Molecolare e Biotecnologie Mediche Università degli Studi di Napoli "Federico II"

Via Pansini 5, IT-80131 Naples (Italy)

E-Mail margherita.ruoppolo@ unina.it 


\section{Introduction}

Diagnostic and therapeutic strategies in genetic and rare metabolic diseases are based on biomarker-driven approaches. Genes, proteins and metabolites are the most frequently used biomarkers. The increase in "omics" technologies offers a great opportunity to dissect a biological system in an unbiased mode. The new concept of personalized medicine relies on global and integrative approaches for patient care. Inborn errors of metabolism (IEMs) are an interesting model for personalized medicine because the deregulated pathways underlying these diseases have been described to some extent.

\section{Inborn Errors of Metabolism}

In 1908, Garrod [1] coined the term "inborn errors of metabolism" to describe genetically determined conditions, which are caused by alterations of a specific enzymatic reaction. IEMs constitute a significant proportion of genetic diseases, particularly in children; however, they are individually rare. Clinical presentations can span from the prenatal period through adulthood and are often nonspecific; therefore, appropriate laboratory tests are essential for formulating a diagnosis [2].

The genetic bases of IEMs are very heterogeneous and can involve genomic rearrangements, deletions or insertions, one or more point mutations. The pathogenesis of IEMs is due to the loss- or gain-of-function of mutant proteins (usually enzymes or transporters), which alter a specific cellular flux. Accumulating upstream metabolites, deficiency of downstream metabolites, or diversion of metabolic flux to secondary pathways can mediate the toxicity of IEM mutations [3].

Because IEMs are linked to a genetic defect, the most frequently used characterization is the identification of the mutated gene and its products. However, the genotype-phenotype correlation is lacking in many IEMs, which highlights the idea that modifying factors can have an impact on the altered pathway, on the metabolic flux as a whole. It is important to keep in mind that these disorders are related to the disruption of specific interactions in a highly organized metabolic network [4]. Thus, it is not easy to predict the impact of a given disruption. Therefore, an integration of scientific skills and methods is needed to assess the altered cellular pathways and the potential interactions with other pathways.

Traditionally, the inherited metabolic diseases were classified as disorders of carbohydrate metabolism, amino acid metabolism, fatty acid metabolism, organic acid metabolism, or lysosomal storage diseases.

Molecular Bases of Inborn Errors of Metabolism

\section{Amino Acid Disorders}

Amino acid disorders are a group of metabolic conditions that impair the synthesis and degradation of amino acids. This causes an increased production of intermediates that become toxic to the central nervous system. The severity of the clinical picture depends on the amino acid involved, the duration of its accumulation and the supervention of other medical complications. Dietary managements are the main treatments for most aminoacidopathies. They aim to prevent accumulation of the substrate to toxic levels and to restore deficiencies of the enzymatic products [5]. Dietary treatment includes natural protein restriction combined with amino acid mixtures lacking the amino acids prior to the metabolic block.

\section{Fatty Acid Oxidation Disorders}

Mitochondrial beta-oxidation is the major pathway for degradation of fatty acids and is essential for maintaining energy homeostasis within the cell. Beta-oxidation is a main energy source for the heart, skeletal muscle, and kidney. Inherited defects are known for most of the gene-encoding enzymes and transporters involved in fatty acid oxidation. Patients have low fasting tolerance showing hypoketotic hypoglycemia that may progress into Reye-like syndrome. Patients may also have skeletal muscle and cardiac disease. In addition, fatty acid oxidation was also shown to have a key role in the pathophysiology of common disorders like insulin resistance, obesity or diabetes. Treatment options for fatty acids oxidation disorders are few [6]. Carnitine supplementation is crucial in patients with primary carnitine deficiency. Patients with other fatty acids oxidation disorders may display secondary carnitine deficiency, which is also treated by carnitine supplementation. Research efforts are employed in this field to design novel therapeutic strategies.

\section{Organic Acidemias}

Organic acidemias or organic acidurias (OAs) are caused by deficiency of enzymatic activities in the catabolism of amino acids, carbohydrates, or lipids [7, 8]. Mono-, di-, or tricarboxylic acids, generally referred as organic acids, accumulate in body fluids. In the neonatal period, OAs lead to toxic encephalopathy, seizures, lethargy, coma, hypotonia, respiratory distress, and poor feeding. For specific OAs, patients can show progressive neurological deterioration, ataxia, Reye syndrome, or psychiatric symptoms. Therapeutic management includes dietary restriction to reduce the substrate of the deficient enzyme 

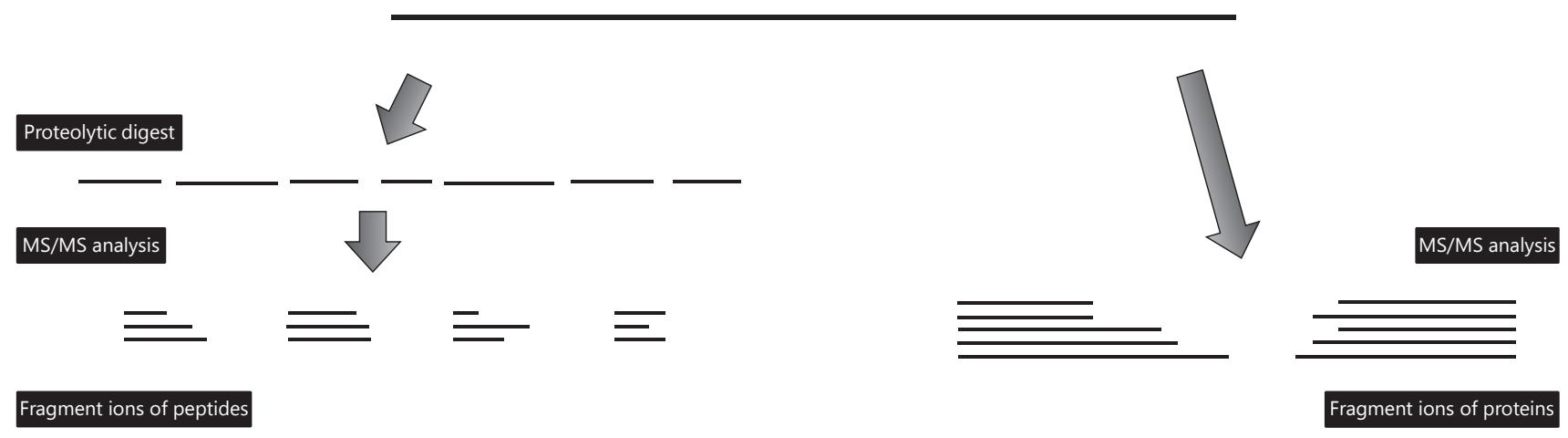

"Bottom-up" proteomics

"Top-down" proteomics

Fig. 1. A schematic view for "bottom-up" and "top-down" proteomics. "Bottom-up" is the most common strategy to identify proteins and characterize their amino acid sequences by tandem mass spectrometry. After proteolytic digestion, peptides are fractionated and further fragmented into mass spectrometer. The "top-down"

and/or supplementation with L-carnitine e/o glycine to increase the excretion of toxic metabolites and/or use of proper vitamins to favor the residual activity of the mutant enzyme, and, finally, liver and/or kidney transplantation [9-11].

Some OAs are comfortably managed if promptly diagnosed and treated, but in some cases brain deterioration is quite common. Formation of the toxic metabolites may in fact occur within the brain [12]. This is particularly true for OAs related to propionate metabolism in which neither diet, vitamin therapy, nor liver transplantation appears to prevent neurological complications [12].

Here, we review the most recent mass spectrometry (MS)-based proteomic and metabolomic strategies used in the diagnosis and in the study of IEMs. Omics strategies play a central role in the postgenomics era of health care. Measurements of metabolites in various body fluids are the current tools for diagnosis of IEMs. MS has in fact facilitated the rapid and economical evaluation of a broad spectrum of metabolites from small samples, including dried blood spots (DBS). This approach has enabled the timely diagnosis of IEMs, thereby facilitating early institution of therapy. approach involves the use of the intact protein whose fragmentation directly occurs in mass spectrometer. The major advantage relies on the possible identification of posttranslational modifications, which are more probably retained along the fragment ions generated from intact protein, rather than protein peptides.

\section{Proteomic Strategies and Applications in IEMs}

Nowadays, proteomics is able to adapt to many different functions and activities taking advantage of the huge improvement in MS techniques and instrumentations to carefully identify thousands of proteins in a biological sample [13]. This makes it attractive in molecular medicine, where it is called clinical proteomics, as a powerful tool able to investigate perturbed pathways causing diseases and discover novel unannotated proteins or biomarkers of disease. A proteomic biomarker is defined as "a specific peptide or protein that is associated with a specific condition, such as the onset, manifestation, or progression of a disease or a response to treatment" $[14,15]$.

The combination of liquid chromatography and electrospray ionization-tandem MS (LC-ESI MS/MS) represents the most effective and used approach for the analysis of proteomes [15].

\section{"Bottom-Up" and "Top-Down" Proteomic Strategies}

The conventional proteomic strategy is called "bottom-up" (Fig. 1). The sample is first subjected to proteolytic digestion and then analyzed by MS; thus, proteins 
are identified by matching the masses of proteolytic peptides or their tandem mass spectra with theoretical in silico digestions performed by bioinformatics tools. Alternatively, new emerging "top-down" approaches do not make use of proteolytic enzymes and propose to analyze intact proteins [16]. While the former approach confirms to be the most robust strategy for protein identification ensuring better separation of peptides compared with proteins [16], the latter aims to identify all the proteoforms of a protein expressed in a sample including genetic mutations, alternative splicing variants, and posttranslational modifications (PTMs) [17]. The identification of PTMs gains a crescent importance in basic and translational research due to their implication in regulating protein structure, function, and localization [18].

\section{Quantitative Proteomics}

The presence or absence of proteins can be useful to characterize in a qualitative fashion full proteomes of cells or tissues. Nowadays, on the other hand, the challenge of quantitative proteomics is to understand how and why protein levels change from a condition to a second one. MS-based quantitative proteomic approaches can be recognized as label-based and label-free (Fig. 2). The first class comprises chemical or enzymatic labeling, including two-dimensional-difference in-gel electrophoresis (2D-DIGE), isotope coded affinity tags (ICAT), isobaric tag for relative and absolute quantitation (iTRAQ), tandem mass tags (TMT), ${ }^{18} \mathrm{O}$-labeling, and metabolic labelling, in particular stable isotope labeling by amino acids in cell culture (SILAC). The strategy contemplates to incorporate stable isotope labels within the peptide, thus introducing an expectable mass difference between the compared conditions detectable by MS. 2D-DIGE makes use of fluorescent dyes coupled to a high-resolution separative method, such as $2 \mathrm{D}$ electrophoresis. These strategies provide the highest accuracy in protein quantitation but they also hold many disadvantages, among which the high cost and possible partial incorporation of labels as well as time expense and operative effort [19].

On the other hand, the application of label-free quantification (LFQ) approaches has become widespread because they seem to overcome all above-mentioned downsides, guaranteeing high-throughput and large-scale protein analysis. The most commonly used LFQ methods can be divided into two groups: (1) spectral counting (SpC) and (2) area under the curve (AUC) or signal intensity measurement. The SpC method provides relative quantification of a protein by counting the number of MS/MS spectra of all the peptides associated with that protein and

Molecular Bases of Inborn Errors of Metabolism

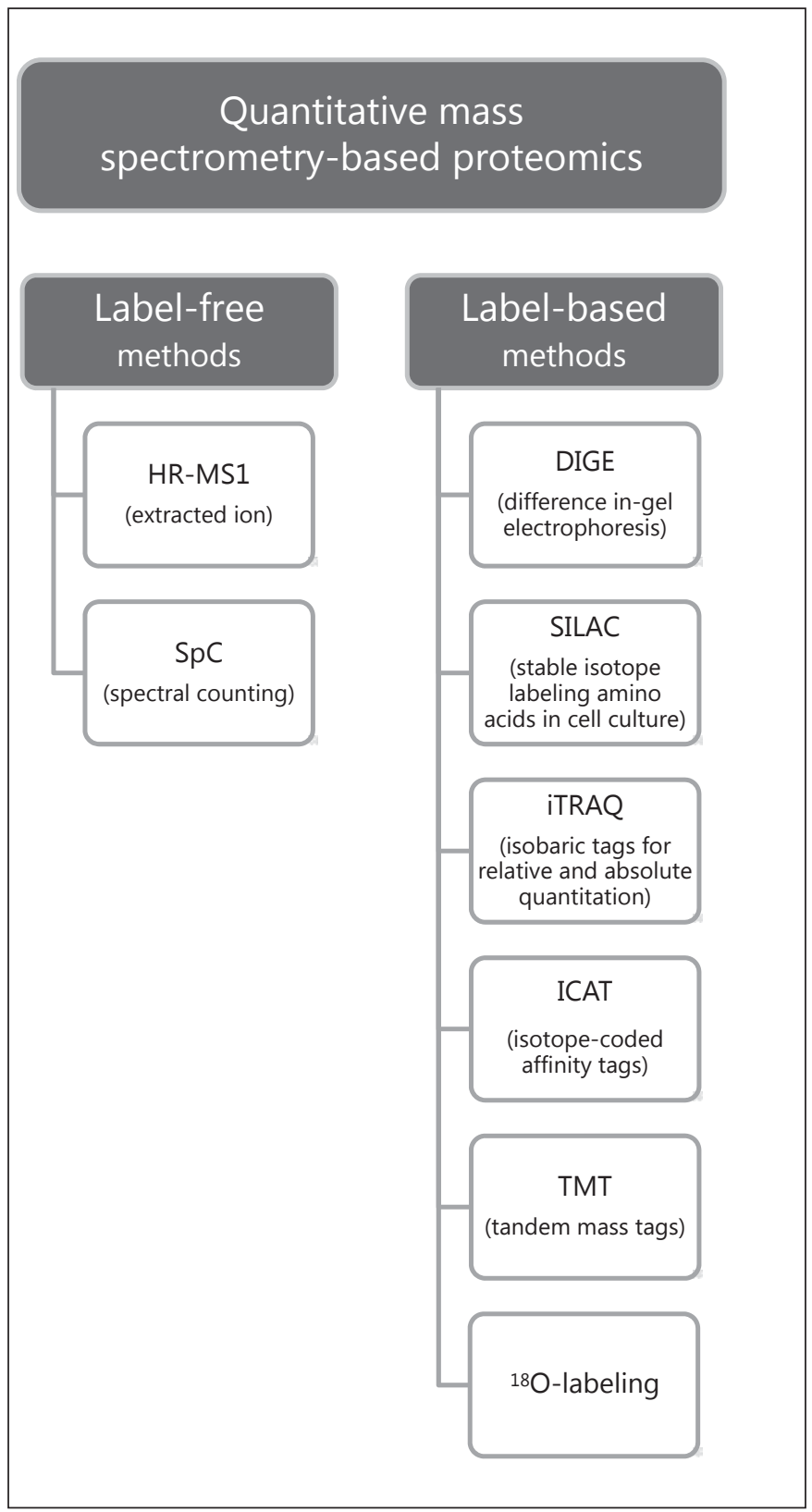

Fig. 2. Graphic representation of quantitative mass spectrometrybased proteomic methods. Quantitative strategies are mainly divided into two groups: label-free and label-based. Label-free methods include spectral counting and high resolution-mass spectrometry (HR-MS1) quantitation by extracted ion. On the other side, label-based methods comprise DIGE, SILAC, iTRAQ, ICAT, TMT, and ${ }^{18} \mathrm{O}$-labeling. These approaches allow the identification and quantification of differentially expressed proteins in 2 or more different samples in comparison, giving the possibility to elucidate molecular pathways in which these proteins are involved. 
correlating it with its abundance in the sample. In the AUC method, protein abundance derives from the extracted ion chromatograms (XIC); peak areas are calculated from precursor ion signal intensity in the full scan MS1 data acquired during an LC-MS/MS run [19-21].

\section{Proteomic Data Interpretation}

One of the most common pitfalls in proteomic experiments is the downstream data analysis combined with interpretation of results. The Human Proteome Organization (HUPO) has developed a set of MS data interpretation guidelines describing the standards for protein identification and acceptability of data. HUPO suggests the need of more stringent criteria with the aim to uniform proteomic community enabling the universal consultation of experimental results [22].

Regardless of the scientific approach used, the big amount of data produced by MS experiments requires the use of advanced software and bioinformatics tools. These are becoming essential in predicting the properties and elucidating structure, interactions, and function of proteins [23].

\section{Urinary Proteomics: Clinical Applications in Genetic} and Rare Metabolic Diseases

The innovation of high-throughput and high-resolution MS-based proteomics has revolutionized molecular medicine, giving the possibility not only to simply identify deregulated proteins but also to frame them within a biological/pathophysiological context. Nevertheless, the preparation of the sample remains the crucial step in proteomic research. Therefore, appropriate refined solubilization, purification, and fractionation strategies are essential for reliability and reproducibility of results [24], as well as numerous biological and technical replicates are required in order to win the statistical significance [24, 25].

Clinical phenotype and the current knowledge of the disease are the starting point for the choice of the sample and the most appropriate experimental procedure. For example, for most of genetic kidney diseases (GKD) urine is the first-rate specimen used for proteomics experiments. Urine represents a cheap and easily available noninvasive specimen, a rich source of information about differentially expressed proteins, peptides and pathways depending on patients' mutated genotype as well as biomarkers of disease [15]. In 2001, Spahr et al. [26] started mapping the urinary proteome identifying 124 proteins; the number of identified proteins grew to over 1,500 in 2006 when Adachi et al. [27] performed an extensive proteomic analysis, up to more than 2,500 proteins when us- ing prefractionation and combining the proteomic data from several urine specimens $[15,28]$. Gel-based or gelfree methods are suitable for urinary proteome analysis. In particular, liquid chromatography-tandem MS and capillary electrophoresis-MS perform rapid separation of protein species in the sample, after preconcentration and enrichment of the low abundant fraction, providing a more accurate analysis especially for peptides and small proteins $(<20 \mathrm{kDa})[13,15,29]$.

Excluding the strictly kidney-related pathologies, the systemic nature of urine has allowed to study the molecular basis and research urinary biomarkers for a large variety of diseases of different origin (in example appendicitis, prostate cancer, tuberculosis, and major depressive disorder) [28].

Increasing attention is being given by researchers to urine-related specimen represented by extracellular vesicles (EVs). Many kinds of EVs have been isolated, among which exosomes, microvesicles, and apoptotic bodies, different from each other by origin, dimension, and protein content [30].

Considering its intricate structure, the glomerulus does not let EVs coming from serum go beyond the areas of the nephron, reinforcing the idea that urinary EVs are mainly derived from renal cells [31]. This is the main reason for considering EVs in research as an excellent source of information in the study of kidney genetic diseases, probably more than the urine itself.

In particular, exosomes originate from all the segments of the nephron through invagination of the plasma membrane forming endosomes, whereupon they are released in the extracellular compartment. They are considered as supramolecular systems containing and transporting proteins [30]. Exosomes contain only 3\% of the total number of urinary proteins; this fact allows researchers to detect the small portion of low-abundance proteins whose mass signal intensities could be covered by the ones produced by high-abundance proteins. In addition, exosomal proteins include a large number of membrane proteins - transporters, receptors, and channels, among others. Consequently, it is of advantage to study these proteins that are widely differentially expressed in order to elucidate molecular mechanisms acting in GKD [30].

For a more detailed description about urine proteomic characterization of GKD, such as glomerular diseases, renal tubular diseases, and polycystic kidney diseases, the reader can refer to specific reviews in that field and references contained therein $[32,33]$.

Urine also represents the substrate to be monitored for onset, manifestation, or progression of many IEMs as well
Costanzo/Zacchia/Bruno/Crisci/Caterino/ Ruoppolo 
Mass spectrometry-based

metabolomics

Untargeted

metabolomics

Identification and quantitation of a full metabolome

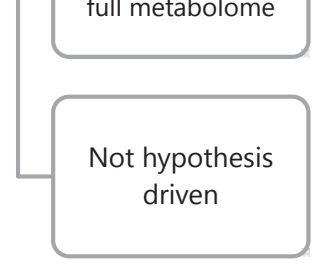

Functional data interpretation
Targeted

metabolomics

Identification and quantitation of a selection of matabolites

Hypothesis driven

Fig. 3. Graphic representation of mass spectrometry-based metabolomic strategies. Two possible approaches can be applied to metabolomics studies and are divided as untargeted and targeted. Untargeted metabolomics allow the identification and quantitation of the whole set of metabolites detectable in a sample. Targeted metabolomics is suitable for the analysis of a limited number of metabolites. The former approach does not follow any hypothesis analyzing data after their acquisition, while the latter establishes a priori hypothesis upon which the choice of metabolites to be investigated is dependent. Interpretation of the qualitative and quantitative metabolomics dataset is the pivotal point of the downstream analysis to correlate data with the biological context.

as diagnosis. However, proteomic strategies are applied to better investigate molecular bases and perturbed pathways in metabolic disorders. In this field, there have been many attempts to elucidate altered pathways in organic acidemias: among these, the proteome of methylmalonic acidemia (MMA) is the most widely investigated. Imperlini et al. [34] discussed the evolution and application of proteomic strategies with emphasis on isolated MMA and MMA with cobalamin deficiency type C. Although proteomic studies have been conducted on fibroblasts or lymphocytes of MMA patients, many of the most promising results have been obtained using liver tissues coming from transplanted patients. The study of the differentially expressed proteins by 2D-DIGE analysis showed their primary involvement in metabolic pathways, such as energy and carbohydrate metabolism, likely to be due to a metabolic adaptation that occurs in liver mitochondria to compensate for the developed dysfunction, a primary trait in MMA patients [34-36].

\section{Metabolomic Strategies and Applications in IEMs}

Metabolomics is an emerging "omics" science involving large-scale study of small molecules, metabolomes and their interaction with the biological system. Metabolites and their concentrations reflect the biochemical activity and collectively provide a "fingerprint" of the linkage between genome and environment. The scientific challenge is represented by the qualitative and quantitative identification of whole metabolomes. The term "metabonomics" was firstly used to indicate the profiling of whole metabolic composition in a living system with simultaneous determination of their changes resulting from multiple environmental conditions as well as genetic background [37]. Finally, "metabolomics" was used to indicate the comprehensive and quantitative analysis of all metabolites in a system [38]. Nowadays, metabonomics and metabolomics are used interchangeably. Metabolomics aims to compare relative differences between biological samples based on their metabolomic profiles.

Results of metabolomic analysis are strictly dependent upon an appropriate experimental design. Sample collection, number of subjects, technological platforms, data mining, and bioinformatics tools are crucial parameters able to maximize the analytical power of metabolomic analysis. A metabolomics study can be performed according to targeted and/or untargeted strategies (Fig. 3). Specific and quantitative targeted metabolomics allows the evaluation of defined metabolites, and it is based on an a priori hypothesis [39]. Nonspecific untargeted metabolomics is performed to determine undefined metabolites, providing a functional fingerprint of the analyzed sample.

\section{Specimens in Metabolomics}

Urine, plasma, or serum samples represent noninvasive or minimally invasive samples. Indeed, urine metabolome is chosen to better describe kidney changes: it is easily and noninvasively collected; blood, plasma, and serum represent minimally invasive samples and are related to systemic changes [40]. A convenient blood sample collection, storage, and transport are obtained using DBS [41]. DBS collection allows obtaining metabolite profiles similar to those from more abundant protein-depleted 


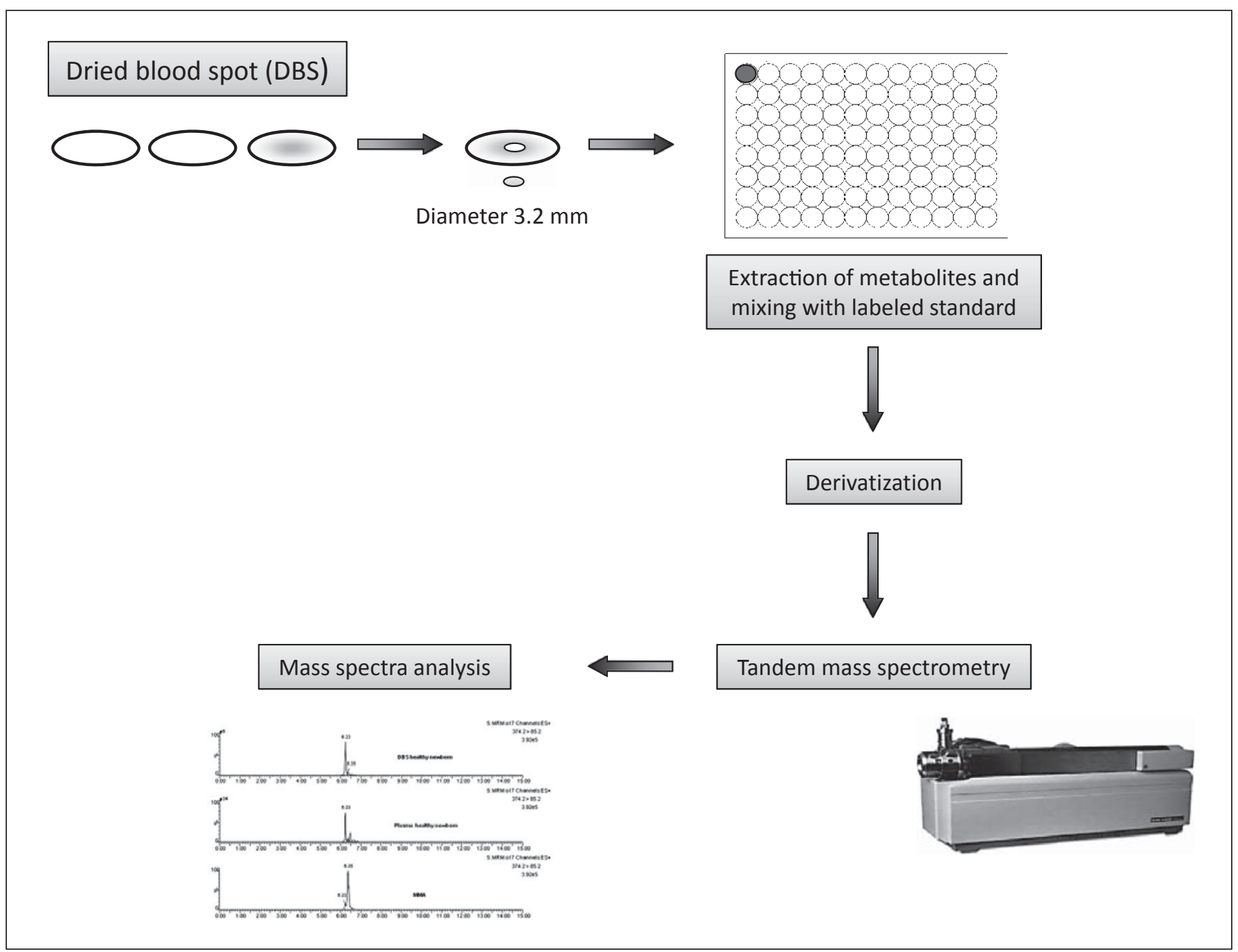

Fig. 4. Flowchart of the methodological strategy for the screening of inborn errors of metabolism. Blood from a prick on the heel of the newborn is collected on dried blood spot (DBS; second-third day of life). This method lets the biological sample be chemically more stable, simplifying delivery modes and allowing easy and

plasma [42, 43]; moreover, it does not require preanalytical steps as sample preparation, storage, freezing, and dry ice transport [44].

\section{Technological Platforms in Metabolomics Analysis}

The different chemical structure and quantitative abundance of metabolites lead to selective, sensitive, and rapid metabolomic methodologies. Due to its high sensitivity and specificity, MS can be used for qualitative and quantitative analysis of many analytes. A big advantage consists in easily coupling MS and separation techniques as chromatography, such as LC-MS, gas chromatography long-term sample conservation. Metabolites are extracted and eluted from the DBS, and suitably prepared for mass spectrometry analysis. The use of neutral loss, precursor ion scan, and multiple reaction monitoring experiments allows quantifying metabolites which are biomarkers of specific inborn errors of metabolism.

(GC)-MS, LC-MS/MS and capillary electrophoresis-MS $[45,46]$. Once the data have been obtained, statistical analysis has a great impact on their biological interpretation. The LC platform provides high sensitivity and selectivity and allows performing the determination of nonvolatile, low- or high-molecular weight compounds, thermally unstable and characterized by a wide polarity range. Reversed-phase LC enables the separation of metabolites with different physicochemical properties. Conversely, hydrophilic interaction LC was used to separate highly polar from ionic molecules. Huge improvements in the chromatographic resolution have recently been obtained 
Table 1. Inborn errors of metabolism identified in our laboratory by dosage of metabolites on dried blood spots by LC-MS/MS analysis

$\begin{array}{lll}\begin{array}{l}\text { Primary markers } \\ \text { (concentration and }\end{array} & \begin{array}{l}\text { Secondary markers } \\ \text { (concentration and } \\ \text { upper cut-off), } \mu \mathrm{mol} / \mathrm{L}\end{array} & \begin{array}{l}\text { Ratio and upper } \\ \text { cut-off }\end{array} \\ \end{array}$

\begin{tabular}{|c|c|c|c|}
\hline \multirow[t]{3}{*}{ Report case 1} & C6 $(0.62,0.14)$ & $\mathrm{C} 10(0.60,0.42)$ & $\mathrm{C} 8 / \mathrm{C} 2(0.245,0.009)$ \\
\hline & C8 $(6.14,0.25)$ & & $\mathrm{C} 8 / \mathrm{C} 10(10.23,2.50)$ \\
\hline & $\mathrm{C} 10: 1(0.51,0.30)$ & & \\
\hline \multirow[t]{3}{*}{ Report case 2} & C6 $(0.64,0.14)$ & $\mathrm{C} 10(0.95,0.42)$ & $\mathrm{C} 8 / \mathrm{C} 2(0.058,0.009)$ \\
\hline & $\mathrm{C} 8(2.39,0.25)$ & & $\mathrm{C} 8 / \mathrm{C} 10(2.50,2.50)$ \\
\hline & $\mathrm{C} 10: 1(0.57,0.30)$ & & \\
\hline \multirow[t]{3}{*}{ Report case 3} & C6 $(0.67,0.14)$ & $\mathrm{C} 10(0.59,0.42)$ & C8/C2 $(0.22,0.009)$ \\
\hline & $\mathrm{C} 8(4.02,0.25)$ & & $\mathrm{C} 8 / \mathrm{C} 10(6.81,2.50)$ \\
\hline & $\mathrm{C} 10: 1(0.43,0.30)$ & & \\
\hline \multirow[t]{3}{*}{ Report case 4} & C6 $(0.34,0.14)$ & $\mathrm{C} 10(0.24,0.42)$ & C8/C2 $(0.04,0.009)$ \\
\hline & $\mathrm{C} 8(0.84,0,25)$ & & C8/C10 $(3.42,2.50)$ \\
\hline & $\mathrm{C} 10: 1(0.13,0.30)$ & & \\
\hline \multirow[t]{3}{*}{ Report case 5} & C6 $(0.34,0.14)$ & $\mathrm{C} 10(0.25,0.42)$ & C8/C2 $(0.02,0.009)$ \\
\hline & C8 $(0.91,0.25)$ & & C8/C10 $(3.66,2.50)$ \\
\hline & C10:1 $(0.19,0.30)$ & & \\
\hline
\end{tabular}

Methylmalonic acidemia with homocystinuria CblC (MMACblC) (OMIM 277400)

$\begin{array}{llll}\text { Report case } 1 & \text { C3 }(3.54,3.18) & \text { C4DC }(0.22,0.49) & \text { C3/C2 (0.12, } 0.14)\end{array}$

Gly $(368,401) \quad$ C3/C16 $(0.64,1.60)$

Isolated methylmalonic acidemia Mut0 (MMAMut0) (OMIM 251000)

$\begin{array}{llll}\text { Report case } 1 & \text { C3 }(8.50,3.18) & \text { C4DC }(0.48,0.49) & \text { C3/C2 }(0.65,0.14) \\ & & \text { Gly }(195,401) & \text { C3/C16(3.25, } 1.60) \\ \text { Report case } 2 & \text { C3 }(32.50,3.18) & \text { C4DC }(0.57,0.49) & \text { C3/C2 }(0.49,0.14)\end{array}$

$\begin{array}{rrr}\text { Cly }(469,401) & \text { C16 (6.90, 1.60) }\end{array}$

Propionic acidemia (PA) (OMIM 606054)

Report case $1 \quad$ C3 $(16.3,3.18) \quad$ Gly $(540,383)$

$\mathrm{C} 3 / \mathrm{C} 2(0.68,0.18)$

$\mathrm{C} 3 / \mathrm{C} 16(6.52,1.90)$

Isovaleric acidemia (IVA) (OMIM 243500)

Report case $1 \quad$ C5 $(5.2,0.43)$

C5/C3 $(52,0.37)$

$\mathrm{C} 5 / \mathrm{C} 4(104,1.96)$

Isobutyryl-CoA dehydrogenase deficiency (IBDHD) (OMIM 611283)

Report case $1 \quad$ C4 $(2.26,0.92)$

Beta-ketothiolase deficiency (BKTD) (OMIM 203750)

$\begin{array}{lll}\text { Report case } 1 & \mathrm{C} 5: 1(0.34,0.11) & \mathrm{C} 5 \mathrm{OH}(1.17,0.4) \\ & \mathrm{C} 4 \mathrm{OH}(2.19,0.45) & \end{array}$

Short branched-chain amino acid deficiency (SBCADD) (OMIM 610006)

Report case $1 \quad$ C5 $(0.81,0.43)$

Formiminotransferase cyclodeaminase deficiency

Report case $1 \quad$ C4 $(4.01,0.92)$

FIGLU $(6.50,1.52)$

3-Methylcrotonyl-CoA carboxylase 2 deficiency (MCC2) (OMIM 210210)

Report case $1 \quad \mathrm{C} 5 \mathrm{OH}(4.09,0.40)$

Cystathionine beta-synthase deficiency (CBSD) (OMIM 613381)

Report case $1 \quad \operatorname{Met}(81,20)$

Molecular Bases of Inborn Errors of

Metabolism 
Table 1 (continued)

$\begin{array}{lll}\begin{array}{l}\text { Primary markers } \\ \text { (concentration and }\end{array} & \begin{array}{l}\text { Secondary markers } \\ \text { (concentration and }\end{array} & \begin{array}{l}\text { Ratio and upper } \\ \text { cut-off }\end{array} \\ \text { upper cut-off), } \mu \mathrm{mol} / \mathrm{L} & \text { upper cut-off), } \mu \mathrm{mol} / \mathrm{L} & \end{array}$

Maternal vitamin $B_{12}$ deficiency

Report case 1

C3 $(5.18,3.18)$

$\mathrm{C} 3 / \mathrm{C} 2(0.145,0.14)$

Report case 2

C3 $(8.30,3.18)$

$\mathrm{C} 3 / \mathrm{C} 16(1.69,1.60)$

Report case 3

C3 $(3.50,3.18)$

$\mathrm{C} 3 / \mathrm{C} 16(2.04,1.60)$

Report case 4

C3 $(7.41,3.18)$

$\mathrm{C} 3 / \mathrm{C} 2(0.11,0.14)$

C3 $(4.17,3.18)$

C3/C16 $(1.69,1.60)$

Report case 5

C3(4.11, 3.18)

$\mathrm{C} 3 / \mathrm{C} 2(0.25,0.14)$

Report case 6

C3 $(4.01,3.18)$

$\mathrm{C} 3 / \mathrm{C} 16(1,92,1.60)$

$\mathrm{C} 3 / \mathrm{C} 2(0.16,0.14)$

$\mathrm{C} 3 / \mathrm{C} 16(1,67,1.60)$

$\mathrm{C} 3 / \mathrm{C} 2(0.23,0.14)$

Report case 7

$\mathrm{C} 3 / \mathrm{C} 16(1.90,1.60)$

$\mathrm{C} 3 / \mathrm{C} 2(0.23,0.14)$

$\mathrm{C} 3 / \mathrm{C} 16(2.12,1.60)$

Maternal carnitine uptake deficiency (CUDmat)

Report case $1 \quad$ C0 $\left(3.18,8^{\mathrm{a}}\right)$

Ctot $\left(13,27^{\mathrm{a}}\right)$

Cbl, cobalamin; C0, free carnitine; C2, acetylcarnitine; C3, propionylcarnitine; C4, butyryl-L-carnitine; C4DC, methylmalonylcarnitine; $\mathrm{C} 4 \mathrm{OH}, 3$-hydroxybutyrylcarnitine; C5, valeryl-L-carnitine; $\mathrm{C5OH}$ hydroxyglutaryl-L-carnitine; C6, hexanoylcarnitine; C8, octanoylL-carnitine; C10, decanoylcarnitine; C10:1, decenoylcarnitine; C16, hexadecanoyl-L-carnitine; Ctot, total carnitine; FIGLU, formiminoglutamic acid; Gly, glycine; Met, methionine.

${ }^{a}$ For this patient only, the reported cut-off refers to the low limit.

by using ultra-high performance LC and two-dimensional-LC techniques [41]. Volatile and thermally stable compounds are traditionally analyzed by GC separation, after derivatization steps. Statistical analysis can be performed by unsupervised methods (principal components analysis) to identify outliers in metabolomics experiments or by supervised methods (partial least squares) to correlate a feature of interest with the entire metabolomic dataset [45-46]. Enrichment analysis is then performed to define biologically meaningful patterns that are significantly represented in the obtained metabolomic dataset. Metabolomic data can be clustered into different networks of metabolic pathways, in which nodes represent experimental and known metabolites [46].

\section{Metabolomics for the Screening of IEMs}

To date, the most successful application of targeted metabolomics analysis is related to the newborn screening of IEMs. The measurement of amino acids and acylcarnitines enables the identification of many IEMs just few days after birth $[47,48]$. If not diagnosed and early treated, most of them might cause mental and/or growth retardation, severe permanent sequelae and, in some cases, death (Fig. 4).

MS-based newborn screening is used not only to make a diagnosis but also to define the optimal therapy and monitor the therapeutic dose. Another important advantage of high-throughput MS-based screening is the very low costs if compared with gene sequencing or SNP profiling [38].

An international collaborative project (http://www. clir-r4s.org) identified 114 biomarkers, amino acids, amino acid ratios, acylcarnitines, and acylcarnitine ratios, to be used in the newborn screening by tandem MS [48, 49]. The marker cut-off values were calculated by the abovequoted collaborative project using approximately 25-30 million normal newborns and 10,742 true positive cases. The range value of a normal population (i.e., normal range), the range value of true positives (i.e., disease range), and the interval between the 99th percentile of a normal population and the 5 th percentile of disease range (i.e., target range) were defined for each biomarker. In order to minimize false negative and false positive results, each biomarker cut-off was set within the target range. 
Table 2. Biomarkers of organic acidemias identified in urine by GC-MS analysis and reference values (mean; 2.5 th-97.5th percentiles)

\begin{tabular}{|c|c|c|c|c|c|}
\hline Metabolite & $\begin{array}{l}\text { Premature infants } \\
<36 \text { weeks } \\
(n=24)\end{array}$ & $\begin{array}{l}\text { Term newborns } \\
>36 \text { weeks } \\
(n=75)\end{array}$ & $\begin{array}{l}\text { Children } \\
<5 \text { years } \\
(n=880)\end{array}$ & $\begin{array}{l}\text { Subjects } \\
5-18 \text { years } \\
(n=511)\end{array}$ & $\begin{array}{l}\text { Adults } \\
(n=60)\end{array}$ \\
\hline Isovalerylglutamic acid & n.d. & n.d. & n.d. & n.d. & n.d. \\
\hline 3-OH-propionic acid & $4.21 ; 1-6$ & $4.26 ; 0-11$ & $4.65 ; 0-13$ & $3.66 ; 0-11$ & $2.19 ; 0-5$ \\
\hline Tiglylglycine & n.d. & $0.11 ; 0-1$ & $0.30 ; 0-2$ & $0.01 ; 0-2$ & $0.01 ; 0-1$ \\
\hline 2-OH-isovaleric acid & $1.00 ; 0-4$ & $0.01 ; 0-0.41$ & $0.01 ; 0-1$ & $0.01 ; 0-3$ & $0.01 ; 0-2$ \\
\hline 3-OH-isovaleric acid & $0.68 ; 0-10$ & $0.23 ; 0-4$ & $2.00 ; 0-11$ & $1.00 ; 0-8$ & $0.01 ; 0-3$ \\
\hline Methylmalonic acid & n.d. & $2.00 ; 0-5$ & $1.29 ; 0-5$ & $1.00 ; 0-3$ & $0.01 ; 0-2$ \\
\hline Propionylglycine & n.d. & n.d. & n.d. & n.d. & n.d. \\
\hline Isovalerylglycine & n.d. & n.d. & $0.15 ; 0-1$ & $0.01 ; 0-1$ & n.d. \\
\hline 2-keto-isovaleric acid & n.d. & n.d. & $0.01 ; 0-0$ & $0.01 ; 0-0$ & n.d. \\
\hline 2-keto-3-Me-valeric acid & n.d. & $0.03 ; 0-1$ & $0.04 ; 0-1$ & $0.11 ; 0-2$ & $0.01 ; 0-1$ \\
\hline 2-keto-isocaproic acid & n.d. & $0.17 ; 0-1$ & $0.20 ; 0-1$ & $0.13 ; 0-1$ & $0.1 ; 0-1$ \\
\hline 2-OH-isocaproic acid & n.d. & n.d. & n.d. & n.d. & n.d. \\
\hline 2-OH-3-Me-valeric acid & n.d. & n.d. & n.d. & n.d. & n.d. \\
\hline 2-Methylcitric acid & n.d. & n.d. & $0.02 ; 0-0$ & $0.01 ; 0-0$ & $0.01 ; 0-0$ \\
\hline
\end{tabular}

Metabolites are quantified as $\mathrm{mmol} / \mathrm{mol}$ creatinine. n.d., not detectable.

Table 1 shows the concentration and upper cut-off values of the primary and secondary metabolic markers, obtained by LC-MS/MS-based expanded newborn screening in our laboratory in the period 2007-2014. Secondtier tests are performed on the DBS by LC-MS/MS analysis for those conditions identified by the same biomarkers, like for example MMA and propionic acidemia, both identified by propionylcarnitine [34]. Data obtained in our laboratory showed that the analysis of amino acids and acylcarnitines in DBS by tandem MS revealed 24 affected newborns among 45,466 infants evaluated between 48 and $72 \mathrm{~h}$ of life (overall incidence 1:1,894).

An interesting advanced procedure to quantify a specific marker for tyrosinemia type I was introduced [49, 50]. The use of succinylacetone as new biomarker was included to avoid false negative results for tyrosinemia type I. Tyrosine, a canonical biomarker of tyrosinemia types I, II, and III, can be transiently increased in many newborns, especially those born prematurely. On the other hand, tyrosine can be within the normal range in reported cases of tyrosinemia type I, thus showing that it is not a good marker for the pathology. The sample preparation was then modified, so that succinylacetone could be extracted as hydrazone derivative and analyzed with other metabolites in a single step [49]. Succinylacetone is in fact produced in case of tyrosinemia type I.

Multiplex MS/MS assays to screen for lysosomal storage disorders in DBS have been developed [51, 52]. It was demonstrated that a single DBS specimen can be used for all the analyses. Newborn screening for lysosomal storage disorders has revealed that late-onset variants of these conditions are unexpectedly frequent and therefore may evade diagnosis. Late-onset variants of these conditions often exhibit a nonspecific phenotype, making it particularly difficult to consider or to justify a request for several single-enzyme activity tests or single-gene analyses.

In case of positive results obtained from LC-MS/MS analysis on DBS, second-level tests are required. Dosages of organic acids in urine constitute the most used secondlevel test. All carboxylic acids and some nitrogen-containing compounds such as pyroglutamate, or amino conjugates like hippurate (benzoylglycine) and glycine derivatives are considered organic acids; short-chain fatty acids are also ascribed to this group of organic substances. Organic acids are water-soluble compounds, usually detectable at the greatest concentration in urine samples. The most complete separation and identification of these metabolites are excellently performed by GC-MS after oximation reaction, liquid extraction, and trimethylsilyl derivatization. The oximation reaction stabilizes 2-keto acids; the trimethylsilyl derivatization improves the thermal stability and volatility of the organic acids. Final identification of the organic molecules is obtained by mass spectra interpretation. Table 2 shows the most common metabolites identified in urine that are biomarkers of a group of IEMs named organic acidemias. 


\section{Conclusions}

Much of our knowledge of protein changes in IEMs arose from differential proteomic analyses using different biological fluids or tissues from patients. These clinical proteomic studies are challenged by specimen availability from patients as well as healthy subjects. Further investigations, including label-free proteomic approaches, could be employed for relative protein quantitation between specimen from patients and healthy controls for their versatility and the required small amount of biological samples.

By a global view of protein variations associated with IEMs, most of the identified differentially expressed proteins are involved in energy metabolism, cellular detoxification, and oxidative stress. Despite these first successful results, the molecular mechanisms underlying most of IEMs pathophysiology still remain to be deciphered. Therefore, we cannot exclude that increased and/or decreased cellular metabolites could regulate epigenetic modifications and modulate the activities of key enzymes, as well as protein PTMs. In this context, MS-based strategies could be a useful platform for the analysis of PTMs, thus indicating which PTMs impact metabolic processes and cellular functions.

Metabolomic analysis of IEMs, derived from extended newborn screening performed using the LC-MS/MS platform, allows quantifying specific biomarkers, which facilitates disease diagnosis, pathogenesis, and therapeutic treatment optimization.

The future challenge in the study of IEM metabolomics is to develop simultaneous targeted and untargeted methods aimed to detect predetermined metabolites with high sensitivity and accuracy, while allowing detection and identification of still unknown metabolites.

\section{Conflict of Interest Statement}

The authors declare that there is no conflict of interests regarding the publication of this paper.

\section{References}

1 Garrod: The Croonian lectures on inborn errors of metabolism, lecture II: alkaptonuria. Lancet 1908;2:73-79.

2 Saudubray JM, Charpentier C: Clinical phenotypes: diagnosis/algorithms; in Scriver CR, Beaudet AL, Sly WS, Valle D (eds): The Metabolic and Molecular Bases of Inherited Disease, ed 8. New York, Mc Graw-Hill, 2001, pp 1327-1403.

3 Lanpher B, Brunetti-Pierri N, Lee B: Inborn errors of metabolism: the flux from Mendelian to complex diseases. Nat Rev 2006;7: 449-460.

-4 Tebani A, Afonso C, Marret S, Bekri S: Omicsbased strategies in precision medicine: toward a paradigm shift in inborn errors of metabolism investigations. Int J Mol Sci 2016;17: 1555-1582.

5 van Vliet D, Derks TG, van Rijn M, de Groot MJ, MacDonald A, Heiner-Fokkema MR, van Spronsen FJ: Single amino acid supplementation in aminoacidopathies: a systematic review. Orphanet J Rare Dis 2014;9:7-21.

6 Houten SM, Violante S, Ventura FV, Wanders RJ: The biochemistry and physiology of mitochondrial fatty acid $\beta$-oxidation and its genetic disorders. Annu Rev Physiol 2016;78: 23-44.

7 Scriver CR, Beaudet AL, Sly WS, Valle D, Childs B, Kinzler KW, Vogelstein B: The Metabolic and Molecular Bases of Inherited Disease, ed 8. New York, McGraw-Hill, 2001.
> Kölker S, Burgard P, Sauer SW, Okun JG: Current concepts in organic acidurias: understanding intra- and extracerebral disease manifestation. J Inherit Metab Dis 2013;36: 635-644.

-9 Knerr I, Weinhold N, Vockley J, Gibson KM: Advances and challenges in the treatment of branched-chain amino/keto acid metabolic defects. J Inherit Metab Dis 2012;35:29-40.

10 Spada M, Calvo PL, Brunati A, Peruzzi L, Dell'Olio D, Romagnoli R, Porta F: Liver transplantation in severe methylmalonic acidemia: the sooner, the better. J Pediatr 2015; 167:1173.

11 Sloan JL, Manoli I, Venditti CP: Liver or combined liver-kidney transplantation for patients with isolated methylmalonic acidemia: who and when? J Pediatr 2015;166:13461350.

12 Kolker S, Okun JG: Methylmalonic acid - an endogenous toxin? Cell Mol Life Sci 2005;62: 621-624.

13 Kentsis A, Monigatti F, Dorff K, Campagne F, Bachur R, Steen H: Urine proteomics for profiling of human disease using high accuracy mass spectrometry. Proteomics Clin Appl 2009;3:1052-1061.

14 Mischak H, Allmaier G, Apweiler R, Attwood $\mathrm{T}$, et al: Recommendations for biomarker identification and qualification in clinical proteomics. Sci Transl Med 2010;2:46ps42.

15 Beasley-Green A: Urine proteomics in the era of mass spectrometry. Int Neurourol J 2016; 20(suppl 2):70-75
16 Gregorich ZR, Chang YH, Ge Y: Proteomics in heart failure: top-down or bottom-up? Pflugers Arch 2014;466:1199-1209.

17 Smith LM, Kelleher NL; The Consortium for Top Down Proteomics: Proteoform: a single term describing protein complexity. Nat Methods 2013;10:186-187.

18 Mann M, Jensen ON: Proteomic analysis of post-translational modifications. Nat Biotechnol 2003;21:255-261.

19 Anand S, Samuel M, Ang CS, Keerthikumar S, Mathivanan S: Label-based and label-free strategies for protein quantitation. Methods Mol Biol 2017;1549:31-43.

20 Neilson KA, Ali NA, Muralidharan S, Mirzaei M, Mariani M, Assadourian G, Lee A, van Sluyter SC, Haynes PA: Less label, more free: approaches in label-free quantitative mass spectrometry. Proteomics 2011;11:535-553.

21 Caterino M, Aspesi A, Pavesi E, Imperlini E, Pagnozzi D, Ingenito L, Santoro C, Dianzani I, Ruoppolo M: Analysis of the interactome of ribosomal protein S19 mutants. Proteomics 2014;14:2286-2296.

22 Deutsch EW, Overall CM, Van Eyk JE, Baker MS, Paik YK, Weintraub ST, Lane L, Martens L, Vandenbrouck Y, Kusebauch U, Hancock WS, Hermjakob H, Aebersold R, Moritz RL, Omenn GS: Human Proteome Project Mass Spectrometry Data Interpretation Guidelines 2.1. J Proteome Res 2016;15:3961-3970. 
-23 Calderón-González KG, Hernández-Monge J, Herrera-Aguirre ME, Luna-Arias JP: Bioinformatics tools for proteomics data interpretation. Adv Exp Med Biol 2016;919:281-341.

24 Wang W, Sue AC, Goh WW: Feature selection in clinical proteomics: with great power comes great reproducibility. Drug Discov Today 2017;22:912-918.

25 Smit S, Hoefsloot HC, Smilde AK: Statistical data processing in clinical proteomics. J Chromatogr B Analyt Technol Biomed Life Sci 2008;866:77-88.

-26 Spahr CS, Davis MT, McGinley MD, Robinson JH, Bures EJ, Beierle J, Mort J, Courchesne PL, Chen K, Wahl RC, Yu W, Luethy R, Patterson SD: Towards defining the urinary proteome using liquid chromatography-tandem mass spectrometry. I. Profiling an unfractionated tryptic digest. Proteomics 2001;1:93107.

-27 Adachi J, Kumar C, Zhang Y, Olsen JV, Mann $\mathrm{M}$ : The human urinary proteome contains more than 1500 proteins, including a large proportion of membrane proteins. Genome Biol 2006;7:R80.

-28 Muntel J, Xuan Y, Berger ST, Reiter L, Bachur $\mathrm{R}$, Kentsis A, Steen H: Advancing urinary protein biomarker discovery by data-independent acquisition on a quadrupole-Orbitrap mass spectrometer. J Proteome Res 2015; 14:4752-4762.

-29 Kalantari S, Jafari A, Moradpoor R, Ghasemi E, Khalkhal E: Human urine proteomics: analytical techniques and clinical applications in renal diseases. Int J Proteomics 2015;2015: 782798 .

-30 Moon PG, You S, Lee JE, Hwang D, Baek MC: Urinary exosomes and proteomics. Mass Spectrom Rev 2011;30:1185-1202.

- 31 Zhang W, Zhou X, Zhang H, Yao Q, Liu Y, Dong Z: Extracellular vesicles in diagnosis and therapy of kidney diseases. Am J Physiol Renal Physiol 2016;311:F844-F851.

- 32 Mullen W, Delles C, Mischak H; EuroKUP COST action: Urinary proteomics in the assessment of chronic kidney disease. Curr Opin Nephrol Hypertens 2011;20:654-661.

- 33 Bonomini M, Sirolli V, Magni F, Urbani A: Proteomics and nephrology. J Nephrol 2012; 25:865-871.
34 Imperlini E, Santorelli L, Orrù S, Scolamiero E, Ruoppolo M, Caterino M: Mass spectrometry-based metabolomic and proteomic strategies in organic acidemias. Biomed Res Int 2016;2016:9210408.

35 Caterino M, Pastore A, Strozziero MG, Di Giovamberardino G, Imperlini E, Scolamiero E, Ingenito L, Boenzi S, Ceravolo F, Martinelli D, Dionisi-Vici C, Ruoppolo M: The proteome of cblC defect: in vivo elucidation of altered cellular pathways in humans. J Inherit Metab Dis 2015;38:969-979.

36 Caterino M, Chandler RJ, Sloan JL, Dorko K, Cusmano-Ozog K, Ingenito L, Strom SC, Imperlini E, Scolamiero E, Venditti CP, Ruoppolo $\mathrm{M}$ : The proteome of methylmalonic acidemia (MMA): the elucidation of altered pathways in patient livers. Mol Biosyst 2016; 12:566-574.

37 Fiehn O: Metabolomics - the link between genotypes and phenotypes. Plant Mol Biol 2002;48:155-171.

38 Wishart DS: Emerging applications of metabolomics in drug discovery and precision medicine. Nat Rev Drug Discov 2016;15:473-484.

39 Oldiges M, Lütz S, Pflug S, Schroer K, Stein N, Wiendahl C: Metabolomics: current state and evolving methodologies and tools. Appl Microbiol Biotechnol 2007;76:495-511.

40 Rifai N, Gillette MA, Carr SA: Protein biomarker discovery and validation: the long and uncertain path to clinical utility. Nat Biotechnol 2006;24:971-983.

41 Gika HG, Theodoridis GA, Wilson ID: Liquid chromatography and ultra-performance liquid chromatography-mass spectrometry fingerprinting of human urine: sample stability under different handling and storage conditions for metabonomics studies. J Chromatogr A 2008; 1189:314-322.

42 Michopoulos F, Theodoridis G, Smith CJ, Wilson ID: Metabolite profiles from dried blood spots for metabonomic studies using UPLC combined with orthogonal acceleration ToF-MS: effects of different papers and sample storage stability. Bioanalysis 2011;3: 2757-2767.
43 Wilson I: Global metabolic profiling (metabonomics/metabolomics) using dried blood spots: advantages and pitfalls. Bioanalysis 2011;3:2255-2257.

44 Kong ST, Lin HS, Ching J, Ho PC: Evaluation of dried blood spots as sample matrix for gas chromatography/mass spectrometry based metabolomic profiling. Anal Chem 2011;83: 4314-4318.

45 Pan Z, Raftery D: Comparing and combining NMR spectroscopy and mass spectrometry in metabolomics. Anal Bioanal Chem 2007:387: 525-527.

46 van den Berg RA, Hoefsloot HC, Westerhuis JA, Smilde AK, van der Werf MJ: Centering, scaling, and transformations: improving the biological information content of metabolomics data. BMC Genomics 2006;7:142.

-47 Scolamiero E, Cozzolino C, Albano L, Ansalone A, et al: Targeted metabolomics in the expanded newborn screening for inborn errors of metabolism. Mol Biosyst 2015;11: $1525-1535$.

48 Scolamiero E, Villani GRD, Ingenito L, Pecce R, Albano L, Caterino M, di Girolamo MG, Di Stefano C, Franzese I, Gallo G, Ruoppolo M: Maternal vitamin B12 deficiency detected in expanded newborn screening. Clin Biochem 2014:47:312-317.

49 la Marca G, Malvagia S, Pasquini E, Innocenti M, Fernandez MR, Donati MA, Zammarchi $\mathrm{E}$ : The inclusion of succinylacetone as marker for tyrosinemia type I in expanded newborn screening programs. Rapid Commun Mass Spectrom 2008;22:812-818.

50 Azzari C, la Marca G, Resti M: Neonatal screening for severe combined immunodeficiency caused by an adenosine deaminase defect: a reliable and inexpensive method using tandem mass spectrometry. J Allergy Clin Immunol 2011;127:1394-1399.

51 la Marca G: Mass spectrometry in clinical chemistry: the case of newborn screening. J Pharm Biomed Anal 2014;101:174-182.

52 Tortorelli S, Turgeon CT, Gavrilov DK, Oglesbee D, Raymond KM, Rinaldo P, Matern D: Simultaneous testing for 6 lysosomal storage disorders and X-adrenoleukodystrophy in dried blood spots by tandem mass spectrometry. Clin Chem 2016;62:12481254.
Molecular Bases of Inborn Errors of

Metabolism
Kidney Dis 2017;3:66-77 DOI: $10.1159 / 000477493$ 